\title{
Partial Superior Movement of Thyroid Cartilage with Minimal Approximation of Arytenoids to Epiglottic Petiole During Swallowing
}

National Cancer Institute

\section{Source}

National Cancer Institute. Partial Superior Movement of Thyroid Cartilage with Minimal Approximation of Arytenoids to Epiglottic Petiole During Swallowing. NCI Thesaurus. Code $\mathrm{C127234.}$

A finding of minimal superior movement of thyroid cartilage with minimal approximation of arytenoids to epig lottic petiole during swallowing. 